\title{
Cocaine affects foraging behaviour and biogenic amine modulated behavioural reflexes in honey bees
}

In humans and other mammals, drugs of abuse alter the function of biogenic amine pathways in the brain leading to the subjective experience of reward and euphoria. Biogenic amine pathways are involved in reward processing across diverse animal phyla, however whether cocaine acts on these neurochemical pathways to cause similar rewarding behavioural effects in animal phyla other than mammals is unclear. Previously, it has been shown that bees are more likely to dance (a signal of perceived reward) when returning from a sucrose feeder after cocaine treatment. Here we examined more broadly whether cocaine altered reward-related behaviour, and biogenic amine modulated behavioural responses in bees. Bees developed a preference for locations at which they received cocaine, and when foraging at low quality sucrose feeders increase their foraging rate in response to cocaine treatment. Cocaine also increased reflexive proboscis extension to sucrose, and sting extension to electric shock. Both of these simple reflexes are modulated by biogenic amines. This shows that systemic cocaine treatment alters behavioural responses that are modulated by biogenic amines in insects. Since insect reward responses involve both octopamine and dopamine signalling, we conclude that cocaine treatment altered diverse reward-related aspects of behaviour in bees. We discuss the implications of these results for understanding the ecology of cocaine as a plant defence compound. Our findings further validate the honey bee as a model system for understanding the behavioural impacts of cocaine, and potentially other drugs of abuse. 
1 Eirik Søvik ${ }^{1,2}$, Naiila Even ${ }^{1}$, Catherine W. Radford ${ }^{1}$, and Andrew B. Barron ${ }^{1}$

2

3 1. Department of Biological Sciences, Macquarie University, Sydney, Australia

4 2. Department of Biology, Washington University, St. Louis, U.S.A.

5

6 Running title: Behavioural effects of cocaine in bees

7

8 Corresponding author: Eirik Søvik

9

10 Department of Biology

11 Washington University in St. Louis

12 Campus Box 1137

13 One Brookings Drive

14 St. Louis, MO 63130-4899

15

16 Telephone: +1 (314) 9353485

17 Fax: $\quad+1(314) 9354432$

18

19 Email: eirik.sovik@gmail.com 
22 Humans and mammals consume drugs of abuse because they make them feel good (Siegel,

23 2005). This presents an unusual paradox (Sullivan, Hagen \& Hammerstein, 2008), since many of

24 the drugs of abuse are naturally occurring plant-derived compounds, and the evolutionary

25 explanation given for the existence of most plant-derived drugs of abuse, is that they evolved as a

26 defence mechanism to deter herbivory (Sullivan et al., 2008). It therefore makes no sense that

27 these compounds should be consumed for their rewarding properties and may even be consumed

28 compulsively. An explanation given for this apparent paradox is that plants evolved to deter

29 herbivores insects (Nathanson et al., 1993), not mammalian ones. This argument assumes that the neurochemical pathways affected by drugs of abuse do different things in these two animal groups such that drugs of abuse are lethal to insects, but rewarding to mammals. By this argument drug reward is viewed as an evolutionary side-effect as mammals are not seen as the co-evolved target of these plant defence compounds. If this explanation is correct, drugs of abuse

34 should not be rewarding to insects.

For a while there was some support for the idea that the neurochemical pathways signalling reward and aversion differed between insects and mammals, however this view is now being revised (Waddell, 2013). The predominant belief was that dopamine, which signals reward in mammalian nervous systems (Schultz, 2007), signalled aversive stimuli in insects (Schwaerzel et al., 2003; Vergoz et al., 2007; Honjo \& Furukubo-Tokunaga, 2009; Nakatani et al., 2009).

However, as more precise genetic tools have become available for studying reward circuitry in insects, it has become clear dopamine plays a role in reward signalling in insects as well (Waddell, 2013). 
45 that a psychostimulant drug can affect the reward system of an insects comes from the finding

46 that following treatment with cocaine, bees are more likely to do a recruitment dance that is

47 highly correlated with perceived reward value of a foraging site (Barron et al., 2009). This

48 indicated that cocaine affected the perceived value of the floral resources collected.

49 Consequently, we investigated the effects of cocaine on reward related behaviours in

50 honey bees. We examined whether honey bees developed a preference for a location in which

51 they had been treated with cocaine, and whether cocaine altered foraging activity. Further, we

52 explored the effects of cocaine on a simple appetitive reflex, sucrose responsiveness (Scheiner,

53 Page Jr \& Erber, 2001, 2004). Lastly, to test if the behavioural effects were limited to reward

54 related behaviours we examined the effects of cocaine on responsiveness to punishing electric

55 shock using the sting extension reflex (Roussel et al., 2009; Giray et al., 2014; Tedjakumala,

56 Aimable \& Giurfa, 2014). We discuss our findings in terms of understanding the actions of

57 cocaine on insects and the implications of this for reconciling the ecological and neurobiological

58 roles of cocaine.

62 Subjects

63 All experiments were performed at Macquarie University, Sydney, Australia. Bees used were of

64 the standard commercially available strains in Australia, and reared according to standard bee

65 keeping practices. For foraging experiments, a colony containing approx. 5000 bees was housed

66 in a $400 \mathrm{~m}^{2}$ flight enclosure.

67

68 Pharmacological treatments. 

(DMF) was applied to the dorsal thorax of bees using a glass microcapillary. This was the same

71 non-toxic dose that increased dance rate in the study by Barron et al. (2009). DMF is a solvent

72 that can penetrate bees' cuticle and allows cocaine to pass into the haemocoel (Barron et al., 73 2007). This method has previously been used for administering cocaine to honey bees (Barron et

74 al., 2009; Søvik, Cornish \& Barron, 2013). As a control, bees were treated with DMF alone in the 75 same manner.

76 For volatilised treatments, freebase cocaine was dissolved in ethanol, and carefully

77 pipetted onto a nichrome wire filament connected to a power source (McClung \& Hirsh, 1998).

78 Ethanol was evaporated from the filament at room temperature. To treat bees, a single bee was

$79 \mathrm{kept}$ in a $50 \mathrm{~cm}^{3}$ airtight container encapsulating the filament. The filament was heated for $10 \mathrm{~s}$

80 and bees were kept in the container, exposed to volatilised cocaine, for one minute. Unlike

81 vertebrates, insects have an open gas exchange system that transports oxygen directly to tissues

82 where it is needed in the gaseous phase, bypassing the haemolymph. Air is taken in through

83 spiracles in the thorax and abdomen, passed through trachea, before gas exchange takes place via

84 tracheoles (Chapman, 2013). This system allows volatilised cocaine to be delivered directly to

85 cells throughout the bee nervous system. As a control, pure ethanol was applied to the filament,

86 allowed to evaporate, and the clean filament was used for treatments using the method outlined

87 above (for details see Søvik et al., 2013). All reagents were supplied by Sigma-Aldrich (St. Louis, $88 \mathrm{MO}, \mathrm{USA})$.

89 Previously we have shown that the pharmacokinetics of these two methods are markedly

90 different (Barron et al., 2009; Søvik et al., 2013), but without measuring the rate cocaine enters

91 and is cleared from the brain following administration it is not possibly to conclusively state how

92 different. 
Effects of cocaine on honey bee foraging preferences.

95 To examine if bees developed a preference for a feeder associated with cocaine treatment, 60

96 individually paint-marked bees were trained to two ad libitum $1.5 \mathrm{M}$ sucrose feeders placed at the

97 closed ends of two $2 \mathrm{~m}$ long tunnels that intersected at a $45^{\circ}$ angle (Fig 1). The walls and floor of

98 the tunnels were solid opaque plastic; the ceiling was covered with mesh. From the perspective of

99 approaching from the hive, the entrance to the left tunnel and the walls surrounding the feeder in

100 the left tunnel were marked with horizontal green and white stripes, the entrance to the right

101 tunnel and the walls surrounding the feeder in the right tunnel were marked with vertical blue and

102 white stripes. The tunnels created two visually distinct and spatially separated environments in

103 which feeders were located. Bee's choice of feeder could easily be assayed visually by observing

104 which tunnel they entered and which feeder they alighted on. The colours blue and green were

105 chosen because bees have distinct photoreceptors for these two colours (Chittka \& Menzel,

106 1992), further, the $90^{\circ}$ difference in orientation of the striped patterns is easily differentiated by

107 honey bees (Frisch, 1971) and was added to make the tunnels even more distinctive. This design

108 was chosen in order to increase the distinctiveness of the two tunnels (i.e. in order to make it as

109 easy as possible for the bees to tell the two tunnels apart). This allowed detecting changes in

110 preference rather than discriminatory abilities.

111 Bees were trained and tested in a five-day protocol. On day one of a trial, bees were

112 trained to use both tunnels by alternating the availability of tunnels every 15 min while

113 progressively stepping a $1.5 \mathrm{M}$ sucrose feeder deeper into each tunnel over a $4 \mathrm{~h}$ period. Bees

114 were released from the tunnel after feeding by lifting the mesh.

115 On day two, bees were further trained to use the tunnels by alternating the availability of

116 the tunnels every $30 \mathrm{~min}$ for $3 \mathrm{~h}$, then simultaneously opening both tunnels to provide bees with a 
117 free choice of feeders for $1 \mathrm{~h}$. During this time the number of visits of each bee to each feeder

118 was recorded. These were converted to a preference index as follows:

$$
\text { Preference index }=\frac{\text { (number of visits to green tunnel }- \text { number of visits to blue tunnel) }}{\text { total number of visits }}
$$

119 This preference index is similar to that used for aversive conditioning by Vergoz et al. (2007), but

120 because individual bees varied in the total number of visits made, we divided difference in visits

121 made by the total number of visits to allow for comparison between bees. At this stage the

122 median preference index was not significantly different from zero (Wilcoxon signed rank test. W

$123=456, \mathrm{p}=0.166, \mathrm{n}=75)$ indicating there was no preference toward either tunnel.

124 On days three and four of a trial, bees had access to the green tunnel only for $2 \mathrm{~h}$ a day,

125 which offered a $1 \mathrm{M}$ sucrose feeder. Bees were randomly assigned to cocaine or control treatment

126 groups. We used the slower topical treatment method so that cocaine would persist in bees'

127 systems for the majority of their time interacting with the tunnels (previous work suggested

128 topical cocaine treatment influenced bee behaviour for approximately $1.5 \mathrm{~h}$ following treatment

129 (Barron et al., 2009), whereas the effects of volatilised treatment appeared to be shorter in

130 duration). Bees were treated with either $1 \mu \mathrm{l}$ DMF containing $3 \mu \mathrm{g}$ cocaine in or $1 \mu \mathrm{l}$ DMF alone

131 on their first visit to the feeder each day.

132 With this assay design bees had more opportunities to visit the green tunnel than the blue

133 tunnel, and therefore had more reinforcing experiences in the green tunnel than the blue tunnel.

134 Thus, expected all bees to develop a weak preference for the green tunnel. However, the aim of

135 this experiment was to test whether cocaine treatment affected the magnitude of the preference

136 for the green feeder.

137 On day five of a trial, all bees were given simultaneous access to both tunnels for $1 \mathrm{~h}$ to

138 test the preference of bees for the different tunnels. The number of visits by each bee to each

139 tunnel was recorded. During the test both tunnels contained empty feeders, and once bees had 
140 reached the end of a tunnel they were released. The number of visits to each tunnel by each bee

141 was converted to a preference index as described. Five replicate trials of this experiment were

142 performed. For analysis data from all trials were pooled.

144 Effect of volatilised cocaine on foraging rate.

145 Previously, Barron et al. (2009) did not find a difference in foraging rate between bees treated

146 with cocaine and controls, using the topical treatment method. As topical treatment is rather slow

147 (Barron et al., 2007) and rate of cocaine delivery to the central nervous system affects the

148 magnitude of behavioural responses (Samaha \& Robinson, 2005), we decided to test if the

149 number of foraging trips was affected following the more rapid volatilised treatment method

150 (Søvik et al., 2013). In a flight cage bees were trained to visit an ad libitum sucrose feeder where

151 they were given individually distinctive paint marks. Bees that returned five times after being

152 marked were caught and treated with $5 \mu \mathrm{g}$ volatilised freebase cocaine or control. We chose $5 \mu \mathrm{g}$

153 as this was the highest volatilised dose previously tested that did cause deleterious motor effects

154 (Søvik, 2013). Bees were assigned to treatment groups randomly. The number of visits treated

155 bees made to the feeder in the 40 min following treatment were recorded. Sucrose concentration,

156 has previously been shown to affect foraging rate in bees (Seeley, 1995), therefore we studied

157 responses of bees to both a low $(0.5 \mathrm{M})$ and a high $(2.0 \mathrm{M})$ sucrose solution.

Effects of volatilised cocaine on sucrose responsiveness.

160 To test if volatilised cocaine affected sucrose responsiveness we used cage-reared bees of known

161 age and social history. Upon emergence, bees were placed in mesh cages $(20 \times 16 \times 3 \mathrm{~cm})$ with

162 ad libitum access to honey. The cages contained eighty bees each, and were kept at $34^{\circ} \mathrm{C}$ for 6

163 days. When bees were 7 days old, they were fastened individually in an $8 \mathrm{~mm}$ tube in a way that

164 prevented the bees from escaping but allowed the proboscis and antenna to move freely 
165 (Bitterman et al., 1983). This method is most commonly used for proboscis extension learning

166 experiments (Felsenberg et al., 2011), but has also been used to measure bees' responsiveness to

167 sucrose (Scheiner et al., 2004). Once harnessed, bees were treated with 0 or $10 \mu \mathrm{g}$ volatilised

168 cocaine and tested for sucrose responsiveness. The $10 \mu \mathrm{g}$ was chosen based on an initial pilot

169 experiment suggesting that this dose was sufficient to elicit increased responsiveness to sucrose

170 (E.S. unpublished data). We repeated this experiment with $0,5,10,20$, or $50 \mu \mathrm{g}$ volatilised

171 cocaine to examine if the effect seen with $10 \mu \mathrm{g}$ was dependent on the cocaine dose used.

172 The sucrose responsiveness test consisted of touching a drop of $10 \%$ sucrose solution to

173 the antennae of bees 3 min after drug exposure, and recording whether or not the proboscis was

174 extended. After the test, bees were tested for their response to water and honey. Bees responding

175 to water, or failing to respond to honey were excluded from the analysis.

Effects of volatilised cocaine on responsiveness to electric shocks.

178 To examine effects of cocaine on responsiveness to electric shock, bees were fastened between

179 two conducting brass plates with a piece of electrical tape (for details see Vergoz et al. 2007).

180 After treatment with $0,5,10,20$ or $50 \mu \mathrm{g}$ volatilised cocaine, brass plates were connected to an

181 electrical supply, and bees were shocked with gradually increasing voltage ( $0.5 \mathrm{~V}$ every 5

182 minutes) from 0-10 V. The first voltage at which a bee extended its stinger (a reflexive response)

183 was recorded for each bee. Testing occurred in front of an extraction fan so no alarm pheromone

184 would linger in the testing room and affect bees yet to be tested (Vergoz et al., 2007).

185 Comparisons between groups were based on $\mathrm{EV}_{50}$ (half maximal effective voltage): the point at

186 which half of all bees in the treatment group extended their stingers.

\section{Results}


Effects of cocaine on honey bee foraging preferences.

191 Repeatedly treating bees with $3 \mu \mathrm{g}$ cocaine in DMF at a sucrose feeder enhanced bees' preference

192 for that feeder in a choice assay when compared to bees treated with DMF as a control (Mann-

193 Witney test: $\mathrm{U}=2185, \mathrm{p}=0.0038$; Effect size: $\mathrm{r}=-0.25$ ). Treating bees with cocaine at a feeder

194 while they were foraging resulted in a greater preference for that feeder in a free-choice test when 195 compared to bees treated with DMF (vehicle control) while foraging at the feeder (Fig 2A).

Effect of volatilised cocaine on foraging rate.

198 Bees treated with $5 \mu \mathrm{g}$ volatilised cocaine once at a $0.5 \mathrm{M}$ feeder made significantly more return

199 visits to the feeder in the 40 min following treatment, than controls $\left(t_{70}=5.0710, p=0.00003\right.$;

200 Effect size: $\mathrm{d}=0.9905$; Fig $2 \mathrm{~B}$ ). Bees treated with cocaine at a $2 \mathrm{M}$ feeder showed no increase in

201 visitations after cocaine treatment $\left(t_{70}=-0.2087, p=0.8353\right.$; Effect size: $d=0.0399$; Fig $\left.2 B\right)$.

202 Demonstrating that bees altered the rate at which they returned to a low quality feeder following 203 volatilised cocaine treatment, but not to a high quality feeder (Fig 2B).

Effects of volatilised cocaine on sucrose responsiveness.

206 Treatment with $10 \mu \mathrm{g}$ of volatilised cocaine increased bees responsiveness to sucrose $\left(\chi^{2}=\right.$ $2076.0268, \mathrm{df}=1, \mathrm{p}=0.0141$; Effect size: $\mathrm{d}=0.6331$; Fig $3 \mathrm{~A}$ ). The effect was dependent on the 208 cocaine dose. Bees treated with 5 and $10 \mu \mathrm{g}$ of cocaine were significantly more responsive to 209 sucrose than controls $\left(\chi^{2}=14.089, \mathrm{df}=4, \mathrm{p}=0.0070\right.$; Fig 3B), while bees treated with 20 or 50

$210 \mu \mathrm{g}$ of cocaine did not differ from controls. The control treatment differed quite markedly between

211 two experiments; however, this is likely because the two experiments were performed at different

212 times of the year. Sucrose responsiveness varies with season and environmental conditions. The

213 important aspect is the different between the cocaine treated bees and the control treated bees in a 214 given experiment. 
Effects of volatilised cocaine on responsiveness to electric shocks.

217 Cocaine affected bees' responsiveness to shock in a dose dependent manner (Fig 3C). We used

218 the $\mathrm{EV}_{50}$ for statistical comparisons. All bees treated with cocaine were significantly more

219 sensitive to electric shock than control treated bees $\left(\mathrm{F}_{4,40}=5.4, \mathrm{p}=0.0015\right.$; Fig $\left.3 \mathrm{C}\right)$. There were

220 no differences between the cocaine treatment groups with the exception of bees treated with 50

$221 \mu \mathrm{g}$ cocaine. The bees treated with $50 \mu \mathrm{g}$ were significantly more sensitive than all other cocaine

222 treated groups $\mathrm{The}^{\mathrm{E} V_{50}}$ of cocaine treated bees $(50 \mu \mathrm{M} \mathrm{EV} 50=2.1 ; 20 \mu \mathrm{M}$ EV50 $=3.5 ; 10 \mu \mathrm{M}$,

$223 \mathrm{EV} 50=2.6 ; 5 \mu \mathrm{M} \mathrm{EV} 50=3.1)$ was lower than in control treated bees $(\mathrm{EV} 50=5.3)$.

\section{Discussion}

227 In two separate experiments we observed that cocaine administration affected aspects of foraging

228 decisions. Cocaine treatment increased the preference for a feeding location, and the rate of

229 visitation at a sucrose feeder (Fig 2). Further, cocaine caused increased responsiveness to sucrose

230 (Fig 3A and B). These findings, as well as those of Barron et al. (2009), lends support to the

231 hypothesis that cocaine alter reward responses across divergent animal groups. However, we also

232 found that cocaine made bees more responsive to electric shock (Fig 3C). Thus, the effect of

233 cocaine is not limited to reward-related behaviours. Rather cocaine altered a range of behavioural

234 responses, all, at least partially, modulated by octopaminergic or dopaminergic signalling. This is

235 consistent with cocaine broadly interfering with octopaminergic and/or dopaminergic signalling

236 in honey bees.

237 Our experiments indicate that cocaine alters the perceived concentration of sucrose in

238 honey bees. Previous studies have shown that bees form stronger associations when rewarded

239 with higher sucrose concentrations compared to lower ones (Loo \& Bitterman, 1992). This can 
240 potentially explain the increased response rate to $10 \%$ sucrose. Interestingly, cocaine only caused

241 bees to increase their visitation rate at the low sucrose concentration feeder. This could be

242 because with high sucrose concentrations, the relative change in perceived sucrose concentration

243 is lower than with low sucrose concentrations.

244 This study provides further support to the bold claim that the neurochemicals modulating

245 reward systems are broadly conserved across diverse animal phyla (Barron, Søvik \& Cornish,

246 2010; Waddell, 2013), and therefore despite certain differences in specific neurochemistry and

247 transporter affinities, diverse reward systems appear susceptible to disruption by the same drugs

248 (Søvik \& Barron, 2013). By 'broad conservation' we do not imply that the reward processing

249 circuitry present in insects and mammals was present in the last common ancestor of these

250 groups, but rather that biogenic amines may have performed functions in the common ancestor

251 that predisposed them to become modulators of reward systems in most animal phyla (Barron et 252 al., 2010).

253 We believe that this is not necessarily contradictory to the ecological function of cocaine 254 as a deterrent compound inhibiting herbivory of the coca plant. Cocaine also enhanced 255 responsiveness to electric shock (Fig 3C), and our previous work has shown cocaine profoundly 256 damaged motor systems, coordination and locomotion in bees (Søvik et al., 2013). Similar

257 findings have been reported for other insects, emphasising the insecticidal properties of cocaine

258 (Nathanson et al., 1993). The effects of cocaine on insects are therefore extremely dose 259 dependent. The rewarding effects reported here were seen at very low doses only. When 260 herbivores ingest plant tissues containing cocaine, they quickly ingest enough to interfere with 261 their motor system, and thus cannot continue feeding (Nathanson et al., 1993).

262 In mammals it is also seen that in recreational drug use, drugs are usually administered in 263 ways that bypass the gut and achieve rapid delivery of a very low and controlled dose to the 
264 central nervous system in order to maximise the hedonic effects while minimising the toxic 265 effects (Hagen et al., 2009).

266 Given the similarities observed in drug responses between vertebrate and invertebrates, it 267 might be possible to use simple invertebrate animals as models for studying aspects of drug 268 reward. While much important work is being done with mammalian models, many other fields of 269 neuroscience have benefitted greatly from the advantages of relatively simple invertebrate model

270 systems (Burne et al., 2011). Previous work with Drosophila has highlighted the importance of 271 circadian regulation (Andretic, Chaney \& Hirsh, 1999; Abarca, Albrecht \& Spanagel, 2002) and 272 LIM-only proteins (Heberlein et al., 2009; Lasek et al., 2010) for the formation of sensitisation. 273 However, invertebrate research has so far not been particularly concerned with drug reward 274 (Søvik \& Barron, 2013). Given the importance of drug reward in human drug use (Siegel, 2005), 275 this should be a key area for future investigations. Honey bees, spend the majority of their time 276 searching out natural rewards in their environments and have a long history as a model organism

277 for studying the neurobiology of natural rewards (Perry \& Barron, 2013). Considering the 278 similarities in responses to cocaine between humans and bees, we can now capitalise on the 279 potential of the honey bee as a simple invertebrate model organism to study drug reward.

\section{Acknowledgements}

283 We would like to thank Falk von Hollen for helping out with the foraging preference

284 experiments, Lea Denneulin and Flavie Rongère for helping with the shock responsiveness 285 experiment. 
Abarca C, Albrecht U, Spanagel R. 2002. Cocaine sensitization and reward are under the influence of circadian genes and rhythm. Proceedings of the National Academy of Sciences of the United States of America 99:9026-9030.

Andretic R, Chaney S, Hirsh J. 1999. Requirement of circadian genes for cocaine sensitization in Drosophila. Science 285:1066-1068.

Barron AB, Maleszka R, Helliwell PG, Robinson GE. 2009. Effects of cocaine on honey bee dance behaviour. The Journal of experimental biology 212:163-168.

Barron AB, Vander Meer RK, Maleszka J, Robinson GE, Maleszka R. 2007. Comparing injection, feeding and topical application methods for treatment of honeybees with octopamine. Journal of insect physiology 53:187-194.

Barron AB, Søvik E, Cornish JL. 2010. The roles of dopamine and related compounds in rewardseeking behavior across animal phyla. Frontiers in behavioral neuroscience 4:1-9.

Bitterman ME, Menzel R, Fietz A, Schäfer S. 1983. Classical conditioning of proboscis extension in honeybees (Apis mellifera). Journal of comparative psychology 97:107-119.

Burne T, Scott E, van Swinderen B, Hilliard M, Reinhard J, Claudianos C, Eyles D, McGrath J. 2011. Big ideas for small brains: what can psychiatry learn from worms, flies, bees and fish? Molecular psychiatry 16:7-16.

Chapman RF. 2013. The Insects: Structure and Function. Cambridge, UK: Cambridge University Press, UK.

Chittka L, Menzel R. 1992. The evolutionary adaptation of flower colours and the insect pollinators' colour vision. Journal of Comparative Physiology A 171:171-181.

Felsenberg J, Gehring KB, Antemann V, Eisenhardt D. 2011. Behavioural pharmacology in classical conditioning of the proboscis extension response in honeybees (Apis mellifera). Journal of visualized experiments $47: \mathrm{e} 2282$.

Frisch K von. 1971. Bees, Their Vision, Chemical Senses, and Language. Itacha, NY: Cornell University Press.

Giray T, Agosto-rivera JL, Stevison BK, Giannoni-guzma MA, Freeman B, Ricci P, Brown EA, Abramson CI. 2014. Ethanol-Induced Effects on Sting Extension Response and Punishment Learning in the Western Honey Bee (Apis mellifera ). 9:1-8. 
Hagen EH, Sullivan RJ, Schmidt R, Morris G, Kempter R, Hammerstein P. 2009. Ecology and neurobiology of toxin avoidance and the paradox of drug reward. Neuroscience 160:69-84.

Heberlein U, Tsai LT-Y, Kapfhamer D, Lasek AW. 2009. Drosophila, a genetic model system to study cocaine-related behaviors: a review with focus on LIM-only proteins. Neuropharmacology 56 Supp1 1:97-106.

Honjo K, Furukubo-Tokunaga K. 2009. Distinctive neuronal networks and biochemical pathways for appetitive and aversive memory in Drosophila larvae. The Journal of neuroscience 29:852-62.

Lasek AW, Kapfhamer D, Kharazia V, Gesch J, Giorgetti F, Heberlein U. 2010. Lmo4 in the nucleus accumbens regulates cocaine sensitivity. Genes, brain, and behavior 9:817-824.

Loo SK, Bitterman ME. 1992. Learning in honeybees (Apis mellifera) as a function of sucrose concentration. Journal of Comparative Psychology 106:29-36.

McClung C, Hirsh J. 1998. Stereotypic behavioral responses to free-base cocaine and the development of behavioral sensitization in Drosophila. Current Biology 8:109-112.

Nakatani Y, Matsumoto Y, Mori Y, Hirashima D, Nishino H, Arikawa K, Mizunami M. 2009. Why the carrot is more effective than the stick: different dynamics of punishment memory and reward memory and its possible biological basis. Neurobiology of learning and memory 92:370-80.

Nathanson JA, Hunnicutt EJ, Kantham L, Scavone C. 1993. Cocaine as a naturally occurring insecticide. Proceedings of the National Academy of Sciences of the United States of America 90:9645-9648.

Perry CJ, Barron AB. 2013. Neural mechanisms of reward in insects. Annual Review of Entomology 58:543-562.

Roussel E, Carcaud J, Sandoz J-C, Giurfa M. 2009. Reappraising social insect behavior through aversive responsiveness and learning. PloS one 4:e4197.

Samaha A-N, Robinson TE. 2005. Why does the rapid delivery of drugs to the brain promote addiction? Trends in pharmacological sciences 26:82-87.

Scheiner R, Page Jr RE, Erber J. 2001. The effects of genotype, foraging role, and sucrose responsiveness on the tactile learning performance of honey bees (Apis mellifera L.). Neurobiology of learning and memory 76:138-50.

Scheiner R, Page Jr RE, Erber J. 2004. Sucrose responsiveness and behavioral plasticity in honey bees (Apis mellifera). Apidologie 35:133-142.

Schultz W. 2007. Behavioral dopamine signals. Trends in neurosciences 30:203-210. 
351 Schwaerzel M, Monastirioti M, Scholz H, Friggi-Grelin F, Birman S, Heisenberg M. 2003. Dopamine and octopamine differentiate between aversive and appetitive olfactory memories in Drosophila. The Journal of neuroscience 23:10495-10502.

354 Seeley TD. 1995. The Wisdom of the Hive. Cambridge, Massachusetts: Harvard University Press.

355

356

357

358

359

360

361

362

363

364

365

366

367

368

369

370
Siegel RK. 2005. Intoxication: The universal drive for mind-altering substances. Rochester, Vermont: Park Street Press.

Søvik E. 2013. Reward processing and responses to drugs of abuse in the honey bee, Apis mellifera. Macquarie University, Australia.

Søvik E, Barron AB. 2013. Invertebrate models in addiction research. Brain, behavior and evolution 82:153-165.

Søvik E, Cornish JL, Barron AB. 2013. Cocaine tolerance in honey bees. PloS one 8:e64920.

Sullivan RJ, Hagen EH, Hammerstein P. 2008. Revealing the paradox of drug reward in human evolution. Proceedings of the Royal Society B: Biological Sciences 275:1231-1241.

Tedjakumala SR, Aimable M, Giurfa M. 2014. Pharmacological modulation of aversive responsiveness in honey bees. Frontiers in behavioral neuroscience 7:221.

Vergoz V, Roussel E, Sandoz J-C, Giurfa M. 2007. Aversive learning in honeybees revealed by the olfactory conditioning of the sting extension reflex. PloS one 2:e288.

Waddell S. 2013. Reinforcement signalling in Drosophila; dopamine does it all after all. Current opinion in neurobiology 23:324-329. 


\section{Figure 1}

Schematic of experimental set-up used for foraging preference experiment.

In the foraging preference experiment, bees were trained to two tunnels. One was blue with vertical stripes while the other was green with horizontal stripes. The difference between the two tunnels was to make it as easy as possible for the bees to tell to two tunnels apart.

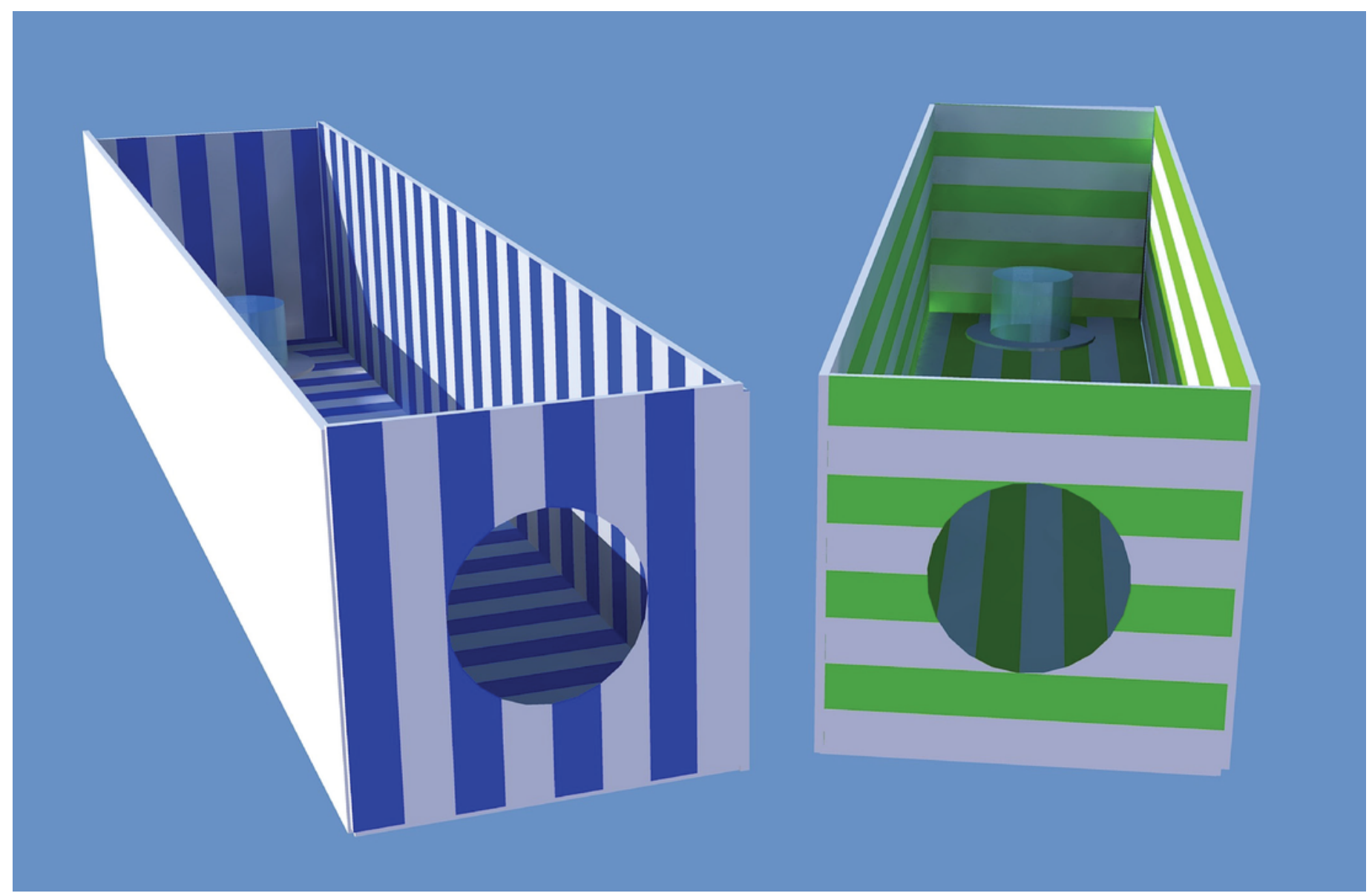




\section{Figure 2}

Foraging behaviour in honey bees following cocaine administration.

A. Scatter plot showing the effect of topical cocaine treatment on preference for the green arm. Each point represents one bee. Dotted lines mark median values for each treatment group. The preference for the green arm was significantly higher for cocaine-treated than control-treated bees (Mann-Witney $U=2185, p=0.0038$ ). B. Effect of volatilised cocaine treatment on visitation rate at a sucrose feeder (error bars represent standard error). Bees treated with volatilised cocaine (grey bars) increased their rate of foraging relative to controls (white bars) when foraging at a $0.5 \mathrm{M}$ sucrose feeder $\left(t_{70}=5.0710, p=0.00003\right)$, but not at a $2 \mathrm{M}$ sucrose feeder $\left(\mathrm{t}_{70}=-0.2087, \mathrm{p}=0.8353\right)$.
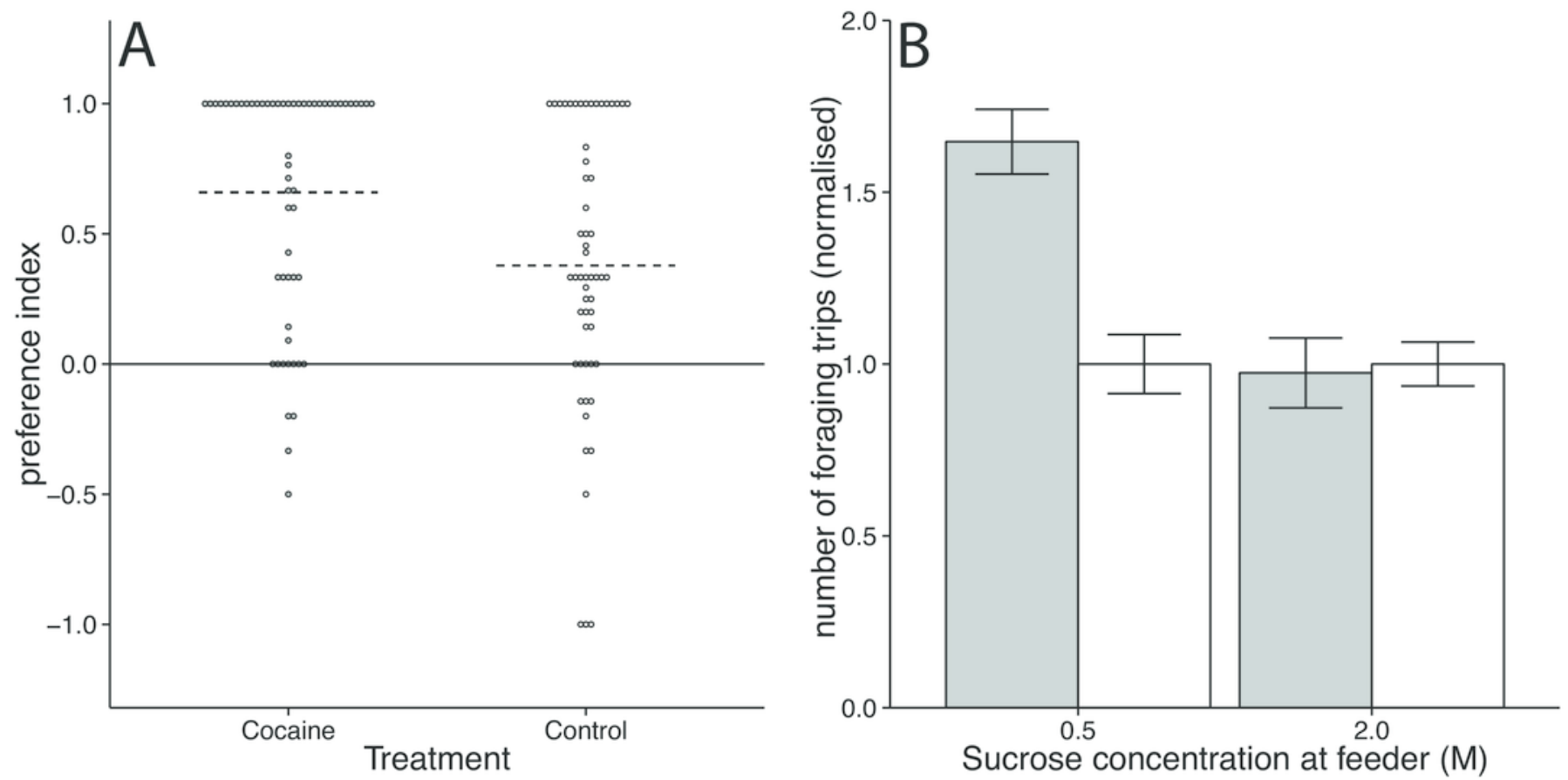


\section{Figure 3}

Behavioural responsiveness following cocaine administration in honey bees.

Proportion of bees responding to $10 \%$ sucrose following treatment with 0 or $10 \mu \mathrm{g}$ of volatilised cocaine (error bars represents standard error and letters denote statistically different groups). There was a significant increase in sucrose responsiveness in bees treated with $10 \mu \mathrm{g}$ cocaine relative to control $\left(\chi^{2}=6.1013, \mathrm{df}=1, \mathrm{p}=0.0135\right)$. B. Proportion of bees responding to $10 \%$ sucrose following treatment with $0,5,10,20$, or $50 \mu \mathrm{g}$ of volatilised cocaine. There was a dose-dependent relationship between cocaine dose and sucrose responsiveness $\left(\chi^{2}=14.089, d f=4, p=0.0070\right)$. C. Shock responsiveness of bees following cocaine administration. Curves are based on weibull distributions of shock responsiveness for each group. Comparisons are based on estimates of $\mathrm{EV}_{50}$ for 40 bees per group $\left(\mathrm{F}_{4,40}=5.4, \mathrm{p}\right.$ $=0.0015)$. Pairwise comparisons found that the $50 \mu \mathrm{g}$ group was different from all other groups, while the remaining cocaine treated groups were different from controls.
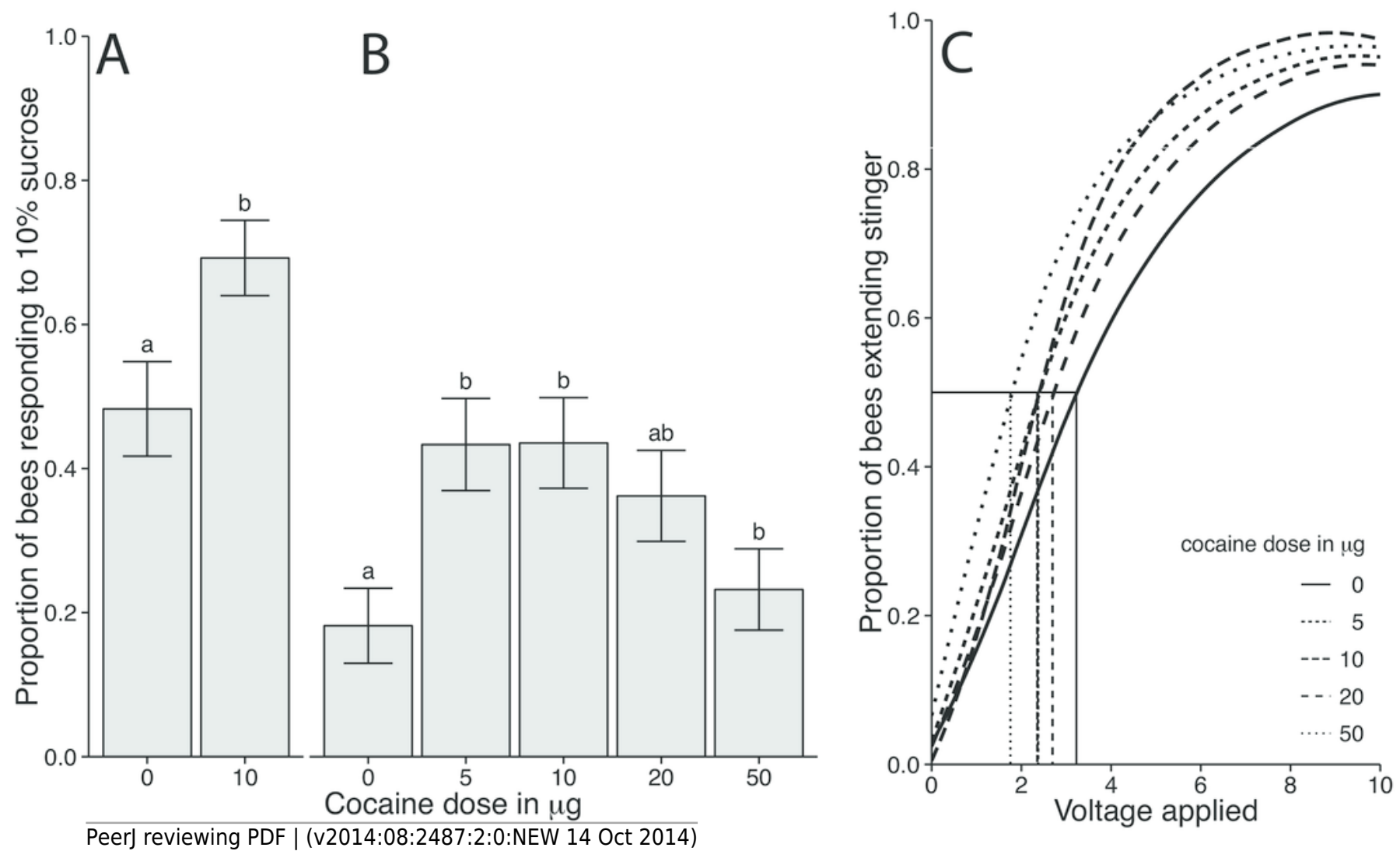